Résumés des conférences et travaux

\title{
Initiation à la littérature sumérienne
}

Michaël Guichard

\section{(2) OpenEdition Journals}

Édition électronique

URL : https://journals.openedition.org/ashp/631

DOI : 10.4000/ashp.631

ISSN : 1969-6310

Éditeur

Publications de l'École Pratique des Hautes Études

\section{Édition imprimée}

Date de publication : 1 octobre 2009

Pagination : 21-32

ISSN : 0766-0677

\section{Référence électronique}

Michaël Guichard, «Initiation à la littérature sumérienne », Annuaire de l'École pratique des hautes études (EPHE), Section des sciences historiques et philologiques [En ligne], 140 | 2009, mis en ligne le 05 octobre 2009, consulté le 06 juillet 2021. URL : http://journals.openedition.org/ashp/631 ; DOI : https://doi.org/10.4000/ashp.631 


\title{
INITIATION À LA LITTÉRATURE SUMÉRIENNE
}

\author{
Chargé de conférences : M. Michaël Guichard
}

Programme de l'année 2006-2007 : Initation à la littérature sumérienne.

La conférence que je dirige, intitulée «Initiation à la littérature sumérienne », s'adresse tout d'abord à des étudiants et des auditeurs qui ont déjà fait au moins deux ans de sumérien et maîtrisent les rudiments de la lecture du cunéiforme.

Nous avons étudié l'année 2006-2007 la singulière histoire du jardinier nommé Šukaletuda. L'histoire fut d'abord découverte par N. Samuel Kramer dans les années 40 et fut annoncée par lui-même dans la revue BASOR, 105 (1947). Celui-ci avait travaillé plusieurs mois en 1946 dans le musée des antiquités d'Istanbul comme représentant de l'University Museum de Philadelphie. Il y copia autant de textes qu'il put (« as many as possible »), tous issus des fouilles américaines datant de 1889-1900. Puisqu'il s'était aussi rendu dans la foulée à Baghdad, ce fut une saison « épigraphique » particulièrement riche («bibliothèque » de Tell Harmal). Il découvrit un fragment de récit sur Gilgameš, des exemplaires d'Enmerkar, de Lugalbanda, des pièces relatives au cycle de Dumuzi, etc. C'est ainsi qu'il annonça que : «Four [fragments] are inscribed with hitherto unknown myth "Inanna and Šukallituda" ». La tablette la plus importante (ni.9721) était divisée en six colonnes dont quatre en bon état : elle est devenue le manuscrit Dn. On retrouve dans les copies de Kramer publiées dans la série turque ISET un trait fébrile, témoignage de l'enthousiasme du copiste, mais un trait un peu difficile à déchiffrer! Apprendre à se familiariser avec les différentes « mains » des épigraphistes (quand on a la chance d'avoir au moins une autographie!) fait partie de l'apprentissage du métier, car pouvoir travailler sur des photos de bonne qualité est aujourd'hui encore assez rare, et le passage au document « réel » est d'ailleurs pour les étudiants une étape déconcertante. La copie a déjà l'avantage pour eux d'être une interprétation qui facilite en principe la lecture.

N. S. Kramer a popularisé sa vision de ce récit dans History begins at Sumer (1959). Il confia à $\mathrm{S}$. Cohen le soin de reconstruire le texte à partir de divers manuscrits incomplets qu'il connaissait. On retrouve sa reconstitution dans la traduction offerte de J. Bottéro et N. S. Kramer dans Lorsque les dieux faisaient l'homme, 1989 (NRF). Enfin le sumérologue allemand K. Volk a reconsidéré l'ensemble du matériel et a proposé une autre reconstitution qu'il a publiée dans la collection Santag en 1995 avec un riche commentaire. Son édition sert aujourd'hui de référence.

Toutefois le texte qui compte 310 lignes comporte encore d'importantes lacunes. La principale se trouve au début puisqu'il manque respectivement 14 et 4 lignes. La fin est particulièrement touchée aussi mais cette situation est partiellement compensée par le caractère répétitif du texte qui permet de combler des plages entières de lacunes. Malheureusement les dernières lignes du poème, peut-être cruciales, sont très endommagées. 
Pour l'heure, le nombre de recensions distinctes répertoriées par K. Volk ne compte pas plus de 11 exemplaires (An, ..., Kn). Neuf d'entre eux proviennent de Nippur et les autres d'Ur (mais seulement sous la forme de petits fragments). C'est donc autant que les manuscrits de Gilgameš et Agga (on en compte aujourd'hui une dizaine), mais beaucoup moins que la Malédiction sur Agade, une œuvre particulièrement populaire, que K. Volk rapproche d'Inana et Šukaletuda. De plus, sa diffusion parait avoir été restreinte à Nippur et à Ur soit un rayonnement qui n'a pas dépassé $150 \mathrm{~km}$. Par ailleurs, les manuscrits dont on dispose sont tous des copies de l'époque paléo-babylonienne (début du deuxième millénaire), ce qui ne présume pas de sa date de composition.

Le poème n'est mentionné que dans un seul catalogue paléo-babylonien retrouvé à Ur, inventoriant 67 titres d'œuvres diverses. On reconnaît dans cette liste quelques sous-ensembles comme la petite collection d'épopées de Gilgameš. Or les œuvres consacrées à la déesse Inana (naturellement l'héroïne d'Inana et Šukaletuda) telles que Inana et Ebih (in-nin-me-huš-a), Ninmešara (dont l'auteur serait Enheduana, fille de Sargon) avec lesquels K. Volk suppose qu'Inana et Šukaletuda a d'étroits rapports, sont isolées les unes des autres. En revanche, le fait que le titre d'Inana et Šukaletuda côtoie le Manuel d'agriculture ( $u_{4}$-ul-la engar) n'est peut-être pas fortuit étant donné le rôle central assigné au jardinier dans le « mythe ».

Inana et $\breve{S}$ ukaletuda, dont le titre ancien était in-nin-me-gal-gal, soit « Maîtresse des grands ME », s'ouvre par un hymne à Inana qui s'en revient du Subartu et de l'Élam, à quoi succède un récit d' « apparition » du premier palmier (thème apparemment incongru, mais une lacune nous empêche de bien comprendre comment il s'articule avec le prologue). L'intrigue débute vraiment à la 1.91 décrivant l'activité du jardinier Šukaletuda et sa rencontre «fortuite » d'Inana endormie et nue sous un peuplier (l'arbre du paradis avant la lettre ?), épuisée par ses récents exploits. Il abuse d'elle sans danger : elle avait détaché sa ceinture où pendaient ses ME (sortes de pouvoirs divins) qui la rendent en principe invulnérable. À son réveil, Inana découvrant le forfait, avec Utu pour témoin, part à la recherche du coupable pour faire justice. Vaines recherches car Šukaletuda, conseillé par son père, se dissimule au sein de la multitude des gens des villes, « ses frères ». Enchaînant plaie sur plaie (notamment le sang dans les puits que Kramer avait rapproché d'une des dix plaies d'Egypte), Inana doit finalement s'en remettre au dieu Enki, dont la demeure se trouve dans l'Abysse (Abzu). Inana ayant adressé sa prière à Enki, en suppliante, Enki s'exclame : « c'est bon! » $\left(i_{3}-s a_{6}\right)$, « ainsi soit-il! » $\left(h e_{2}-a m_{3}\right)$.

Inana retrouve alors Šukaletuda, lui annonce qu'il doit mourir, mais néanmoins lui promet que son nom ne sera jamais oublié :

Un jeune musicien fera à ton sujet une douce mélodie dans le palais royal.

Le pasteur l'entonnera pour le plaisir au (son du) glou-glou de la jarre (de lait).

Le jeune berger apportera ton nom jusque dans sa bergerie.

On apprend enfin que le jardinier (mort, mais destiné à la gloire !) s'est vu assigné une demeure éternelle dans un lieu appelé $e_{2}$-[gal $]$-eden-na « palais-de-la-steppe » lieu invoqué dans les incantations où sont relégués les démons bons ou mauvais.

Les dernières lignes sont perdues mais le texte se clot par une courte et classique doxologie « [Jeune Femme] Inana, louange (à toi)! » 
Par ces longues répétitions (était-ce un texte destiné à être porté en musique ou/et récité avec légèreté comme l'écoulement saccadé d'un bouteille ?), ce texte est certes impropre à une lecture pour soi. Pourtant la qualité de l'œuvre se laisse saisir dans la force expressive de quelques passages-clé.

Tout à fait impressionnant nous a paru, par exemple, le moment où Šukaletuda qui s'applique à cultiver des bandes de terre est pris dans une violente tempête de sable qui l'aveugle d'abord, - peut-être ce vent du sud-est (le Sharqi) dont les bourrasques sont réputées si terribles :

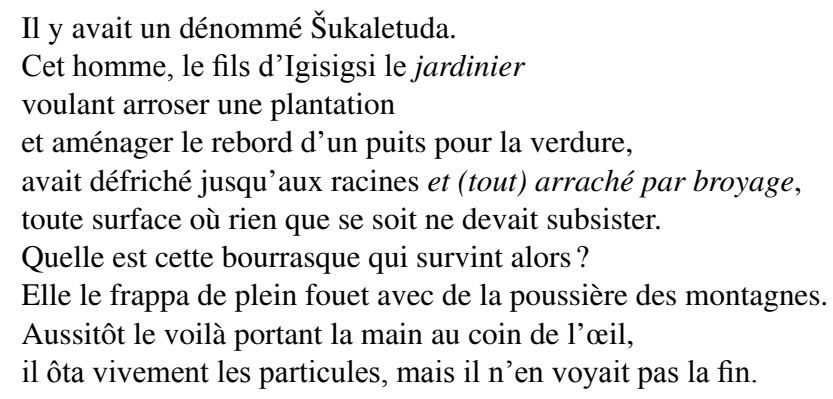

Il faut supposer ici que le vent est brusquement tombé laissant place à la vision mystérieuse d'un ciel limpide :

Il baissa le regard et se mit à contempler ceux du haut-ciel d'Orient,

(et) il leva le regard et se mit à contempler ceux du haut-ciel d'Occident.

(Cela) lui permit d'entrevoir un spectre solitaire qui se mouvait,

Il reconnut un dieu solitaire qui se mouvait.

Il vit l'ordre (divin) tel qu'il devait s'accomplir,

Il eut la vision du destin (fixé) par les dieux.

Il semble ainsi que tout ce qui a lieu ensuite, le viol d'Inana, les malheurs de Sumer et la fin de Šukaletuda, faisait partie d'un plan divin. Le corps céleste qui se déplace dans le ciel est une allusion à Inana dans son aspect astral. Quant à Šukaletuda (présenté au début comme un rustre d'après la manière dont il travaille selon une lecture de P. Attinger), s'il a bien sûr pu agir ensuite en connaissance de cause (soutenu par cet omen et attiré par la gloire), il risque plutôt de ne pas avoir saisi le sens de sa vision d'autant que généralement l'homme avait besoin d'un interprète pour saisir la signification des messages divins et lorsqu'il vit Inana, il fut surtout guidé par son instinct. Le passage servirait surtout à avertir l'auditoire que les événements rapportés ensuite n'étaient pas gratuits, mais obéissaient aux desseins des dieux.

Cette histoire a en effet quelque chose de scandaleux pour plusieurs raisons : Šukaletuda viole une déesse (ce qui en soi est déjà quelque chose!) dans son sommeil et rien en lui n'évoque une figure particulièrement héroïque. Il est même de ce point de vue un anti-héros, remarquable de lâcheté. En se fondant dans la foule pour se dissimuler, il provoque une série de catastrophes pour les hommes ! Par ailleurs, même s'il reçoit comme punition la mort (ce qui à vrai dire est le lot des hommes), il est récompensé par la gloire qui est tout ce que recherchaient les puissants de ce monde. Enfin la déesse Inana est décrite comme une divinité imprudente et impuissante à retrouver le coupable qu'elle veut châtier. Son portrait est donc peu flatteur. 
K. Volk a proposé de trouver en arrière plan de cet étonnant récit, un motif historique, supposant que le moment de sa composition serait en rapport avec le déclin ou l'effondrement de l'empire d'Agade, dont la divinité tutélaire était Inana. On y retrouverait une transposition du souvenir des inutiles répressions de l'empire face à la résistance locale de Sumer. Inana et Šukaletuda servirait en outre de contrepartie aux grands récits guerriers d'Inana tels qu'Inana et Ebih. On pourrait aussi retrouver dans l'histoire une sorte de satire de la légende de Sargon. D'après une des traditions qui circulait concernant l'exceptionnelle carrière politique de ce dernier : il aurait été à l'origine ce jardinier dont se serait éprise Inana. Suite à cette élection divine, le succès n'aurait plus quitté Sargon, le fondateur de l'empire. Il serait alors tentant de voir dans Šukaletuda un anti-Sargon. Non seulement l'union entre Inana et le jardinier est stérile mais elle devient source de malheur. Inana et $\breve{S} u k a l e t u d a$ serait la revanche imaginaire des gens de Sumer (la littérature comme arme des vaincus!).

À cette lecture « historisante », qui s'inspire d'une démarche déjà initiée par C. Wilcke et n'entraîne pas forcément l'adhésion de tous, on peut opposer celle B. Alster qui veut souligner le caractère ludique de l'œuvre. La définition du genre d'Inana et Šukaletuda est aussi un sujet de discussion. Ce ne serait pas une épopée mythologique comme le propose K. Volk, mais un hymne (humoristique) construit sur une fable mythologique, selon B. Alster.

Enfin, nous avons relevé que sur le plan purement mythologique Inana et Šukaletuda possède une intrigue vaguement parallèle à celle d'Adapa dont nous savons qu'il existe un précurseur sumérien (encore inédit jusqu'à ce jour). Ces deux «narrations » nous situent aux origines des temps, elles traitent de la tension entre deux pôles divins, Enki/ Inana, dans notre histoire, Enki/An dans Adapa. Un homme (Šukaletuda/Adapa), doté d'une maîtrise technique (l'art du jardinage/la sagesse et l'art de la pêche pour les offrandes) est confronté à une divinité dont il « abuse » ou qu'il « maîtrise » (Inana est violée, le vent-Sutu voit ses aîles coupées et An s'en trouve lésé). Face à ce drame Inana et An sont impuissants. Cela conduit à une catrastrophe terrestre (les plaies d'un côté, la sécheresse et la mort de l'autre). Finalement les héros connaissent un destin funeste (l'immortalité échappe à Adapa), mais à cause de leur exploit (Adapa est crédité d'être monté au ciel) acquièrent une reconnaissance divine. À chaque fois la supériorité d'Enki est mise à l'honneur. Dans Adapa, grâce à Enki, l'humanité reçoit une leçon et un certain savoir magique tandis que dans Inana et $\breve{S} u k a l e t u d a$, l'intelligence créatrice d'Enki l'emporte sur la violence triomphante incarnée par Inana. Mais Inana, finalement magnanime, s'en trouve grandie.

Quant à Šukaletuda, il est honoré lui aussi car l'auditoire s'est reconnu en lui, lui tout instrument du destin des dieux (et d'Enki probablement) qu'il ait été, a fait l'amour à une grande déesse !

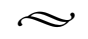

Programme de l'année 2007-2008 : Initiation à la littérature sumérienne.

Cette année nous avons étudié un récit épique fameux de la littérature sumérienne, Gilgameš et Agga (ou Ak[k]a), une des pièces du « cycle » des aventures du roi d'Uruk. 
De tous les récits sumériens qui s'attachent à la geste de Gilgameš (en sum. Bilgames) celui-ci est sans doute l'un des plus étudiés (C. Wilcke, Mél. Römer, 1998, p. 457; les éditions principales sont celles de ETCSL [le site internet d'Oxford], D. Katz, LOT 1, 1993 et W. Römer, AOAT, 290 [1990]). C'est la plus courte des œuvres et elle a pu être intégralement reconstituée (l'exemplaire de la collection Schøyen est toujours inédit, mais nous avons pu néanmoins en étudier la face grâce à un cliché disponible sur internet). Elle est pourtant loin d'être facile à interpréter tant au plan grammatical que poétique (J. Cooper, JCS, 33 [1981] ; C. Wilcke, Mél. Römer, 1998). Comme souvent dans le genre épique sumérien, il y a très peu d'actions, des passages entiers sont répétés (J. Cooper, JCS, 33 [1981], p. 226), la force des images (qui nécessitent une véritable herméneutique) qui se succèdent y joue un rôle privilégié et y résonnent assonnances et allitérations (T. Marik, ArOr, 66 [1996]), phénomène discutable toutefois puisqu'il se heurte au problème du choix de la valeur phonétique des logogrammes tel que, par exemple, le signe $\mathrm{PU}_{2}$ à lire $t u l_{2}$ ou $p u_{2}$ (avec cette dernière lecture attestée par la série lexicale $A=n \bar{a} q u$ [36:1] par opposition à $t u l_{2}=i s s \hat{u}$ «puits à argile » [37:1], qui produit une eau boueuse, le jeu de correspondance avec le verbe til serait perdu). Des dictons ou des proverbes ont été incorporés dans la trame narrative ce qui, avec l'oubli de cette sagesse académique, obscurcit pour nous beaucoup le texte (cf. en dernier lieu B. Alster, CUSAS, 2 [2007], p. 118). Sur les 114 lignes que compte le récit, plus de la moitié appartient au discours direct, une des techniques poétiques préférées de la littérature sumérienne pour faire progresser l'action (T. Marik).

Ce récit présente la particularité, quoique se déroulant dans une époque légendaire, de ne pas faire intervenir les divinités directement; seuls sont mentionnés Ištar, à un moment décisif, et An de manière plus indirecte et conventionnelle. Aussi a-t-on été tenté d'y voir pratiquement un texte historique, d'autant qu'il reflèterait bien l'ambiance du Dynastique Archaïque avec l'évocation de l'hégémonie de Kiš qui donna naissance au milieu du III $^{\mathrm{e}}$ millénaire au titre prestigieux de « roi de Kiš » (J. Cooper, JCS, 33 [1981], p. 229). D. Katz distingue le niveau légendaire, très ancien, de la composition littéraire qu'elle situe à la fin du III ${ }^{\mathrm{e}}$ millénaire. En réalité, l'histoire ellemême - c'est-à-dire le déroulement des événements - est « éclipsée » par le style formulaire qui prédomine et structure l'œuvre (T. Marik), ce qui est courant dans la poésie orale ou écrite (J. Cooper, JCS, 33 [1981], p. 227). Mais la structure (par cercles selon D. Katz) est porteuse de sens, comme les parallélismes et les répétitions.

Quoi qu'il en soit, Gilgameš, qui est présenté dans l'épopée akkadienne comme aux deux tiers divin, est un héros dont la puissance est ici aussi sentie comme surnaturelle. Cependant D. Katz croit que son image est celle d'un homme ordinaire $(R A, 81$ [1987], p. 105), P. Michalowski trouve incertaine la nature exacte de la figure sumérienne (CSMS Bulletin, 16 [1988]).

Après avoir essuyé le refus des Anciens de s'opposer à l'ultimatum d'Agga de Kiš que celui-ci a transmis à Uruk par ses messagers, Gilgameš obtient le soutien inconditionnel et exalté des jeunes de sa ville. La ville d'Uruk, dont Gilgameš est le seigneur (en), est alors assiégée par l'armée de Kiš. Il suffit à Gilgameš d'apparaître sur le haut des remparts (ce qu'il ne fait pas tout de suite) pour causer la confusion (?) des assiégeants - le tout s'accompagnant d'une tempête recouvrant le monde entier - tandis que le roi de Kiš est capturé au milieu de son armée. Mais le héros le libère après 
avoir reconnu la dette contractée à son égard : Agga l'aurait autrefois recueilli et placé à son service (cela dit, ce passé n'est évoqué que par métaphores). L'histoire se termine ainsi par un acte de pardon qui révèle un trait essentiel de la personnalité du héros qui se retrouve dans Gilgameš et Huwawa (J. Cooper, JCS, 33 [1981], p. 230). Il est vrai que son attitude est loin d'être désintéressée (D. Katz, LOT 1).

Avant d'aborder directement le «poème épique », nous avons cherché à partir d'autres documents à retrouver les traces de l'ancienneté tant du « texte » que du thème qu'il traite. Se pose en effet la question de savoir si les versions paléo-babyloniennes viennent d'un récit précurseur du Dynastique Archaïque, période où se situe précisément l'action de Gilgameš et Agga. Disons d'emblée qu'il n'est guère possible de remonter au-delà de l'époque d'Ur III (vers 2100). Le mot de D. Katz (RA, 81, p. 106) « more fiction than facts » résume bien la situation mais n'empêche pas une recherche sur les racines de la fiction dans l'histoire.

Nous avons pour cela étudié des extraits de plusieurs versions de la Liste royale sumérienne, en particulier le précurseur qui date du règne de Šulgi (2093-2046) récemment publié (P. Steinkeller, Mél. Wilcke, 2003). Le caractère secondaire des légendes qu'on trouve dans cette liste, déjà soupçonné par T. Jacobsen, se trouve confirmé par cette version ancienne (plutôt aride) qui malheureusement est incomplète. Or, à l'époque paléo-babylonienne, plusieurs recensions comportant d'importantes divergences coexistaient. Les traditions sur Uruk semblent se contredire. Si pour Jacobsen, les motifs légendaires, quoiqu'ajoutés tardivement à la Liste royale sumérienne, étaient certainement anciens, rien n'empêche non plus qu'ils représentent des « créations » récentes élaborées à partir du schéma historique construit artificiellement par ces mêmes listes.

Toutefois, l'ancienneté du rôle de « libérateur » de Gilgameš est confirmée par une inscription du roi d'Uruk Utu-hegal (vers 2100) qui raconte comment il s'est arrogé le pouvoir à Uruk, a bouté l'envahisseur Gouti hors de Sumer et a fait revenir la royauté à Sumer (RIME 2.13.6.4). Nous avons relu ce document, dont l'authenticité peut, il est vrai, être sujette à caution (H. Sauren, $R A, 61$ [1967]), non seulement parce que seules deux copies paléo-babyloniennes nous sont parvenues mais aussi à cause de son caractère « romancé ».

Dans un passage clé, Utu-heg̃al se vante d'être directement soutenu par Gilgameš, thème plutôt rare dans ce genre d'inscription. En route vers l'ennemi, au camp d'E'Iškura, une étape après Uruk, Utu-heg̃al exprime ses prétentions par un discours légitimateur, teinté de «nationalisme » (1. iii, 23-iv, 8) :

Il déclara aux « citoyens » de sa ville :

— Enlil m'a livré le Gutium. Ma reine Inana est mon alliée. Dumuzi-Ama-ušumgalana a dit : «C'est mon affaire! » Gilgameš, fils de Ninsumun, m'a offert d'en être le dirigeant (maškim).

Il inspira de la joie chez les « citoyens » d'Uruk (et) les « citoyens » de Kulaba. (Les gens de) sa ville comme un seul homme le suivirent.

Utu-heg̃al a un point commun avec Gilgameš, il doit obtenir le soutien des habitants d'Uruk pour lancer sa campagne contre les Goutis, même si le genre de l'inscription atténue cette réalité : son autorité est définie par le terme maškim, qui dési- 
gne quelqu'un qui a une délégation royale et accomplit à la place du monarque des tâches religieuses, juridiques ou militaires (W. Sallaberger, $O B O$ 160/2). Dans le cas présent, c'est le divin Gilgameš qui lui a transmis ce pouvoir directement. Les gens d'Uruk (ici les dumu-Unu-ga partis à la guerre, donc sans doute l'équivalent des g̃uruš du poème épique) ne font qu'enregistrer le fait. On a relevé le parallélisme entre les lignes iii, 23-iv, 8 de l'inscription d'Utu-hegal et $G$. et $A$. 15-41 quoique les rapports soient inversés : le chef d'Uruk légitime sa campagne $\rightarrow$ enthousiasme du peuple // le peuple légitime le seigneur d'Uruk $\rightarrow$ enthousiasme de ce dernier. Il est très probable que la représentation de la guerre avec le Gutium s'inspire du drame légendaire de la lutte d'Uruk contre l'hégémonie de Kiš. Mais si tel est le cas, le traitement impitoyable réservé au roi des Goutis à la fin de la campagne serait une référence à une version « dure » de Gilgameš et Agga. Un autre problème vient aussi du rôle de précurseur qu'une tradition attribue à Dumuzi qui « fut fait prisonnier par la main d'Enmebaragesi » (J. Klein, Mél. Sigirst, 2008, p. 77) et que manifestement le scribe de l'inscription d'Utu-hegal ne devait pas ignorer non plus pour mentionner aussi Dumuzi comme défenseur (malheureux semble-t-il) d'Uruk.

Nous sommes finalement arrivés à la conclusion que l'inscription d'Utu-hegal ne pouvait pas prouver l'existence à cette période du récit de Gilgameš et Agga dans la seule version qui nous est parvenue.

Ensuite nous avons étudié un extrait de l'hymne O de Šulgi qui, malgré ses lacunes, est une pièce essentielle du dossier. Šulgi, le roi d'Ur, se vante d'entretenir des rapports fraternels avec Gilgameš qu'il présente comme son ami et son frère, ce qu'il fait aussi avec Utu dans l'hymne A. La scène les représente tous les deux faisant l'éloge de leurs actions respectives. Nous avons tenté une traduction provisoire du texte avec l'aide de l'édition de J. Klein (AOAT 25) et de diverses approches dont en dernier lieu celle de A. George, The Babylonian Gilgamě̌ Epic, 2003 :

Le Pasteur Šulgi, fils de Nin-sumun,

30 Le $[\ldots]$ qui est parfait pour $[\ldots]$

[Qui n'a de tolérance aucune] pour les mensonges de ses [ennemis],

32 [Le héros] Bilgames, le seigneur de Kulaba

Le laissa entrer dans son [palais (?)].

$34[($ Šulgi)...] lui rapporta des nouvelles de $[\ldots]$ des contrées.

C'était $[\ldots]$ de son palais.

36 C'était $[\ldots]$ des pays.

[Alors (Bilgames)] le regarda comme au travers d'un roseau fendu.

38 Depuis le jour où le destin du pays fut décidé,

Et depuis que la semence des êtres vivants est apparue,

40 Un roi s'étant (enfin) présenté devant son alter ego avec éclat, Ce jour-là donc, Bilgames, le seigneur de Kulaba,

42 (et) Šulgi, le pasteur juste de Sumer - (qui était là) à ses pieds immaculés - s'entretinrent l'un avec l'autre.

44 Pour que la gloire éternelle leur soit rendue, Pour qu'elle soit transmise dans les jours lointains,

46 Qu'elle ne tombe pas dans l'oubli dans les années futures, Eux-mêmes de leur puissants actes héroïques Firent l'éloge bien fondé. 
Šulgi, le pasteur juste de Sumer,

50 Exalta pour sa puissance

Son frère et ami Bilgames

52 Et lui rendit hommage en évoquant ses actions héroïques :

- (Toi) le plus fort au combat, qui a anéanti tant de cités,

54 Le massacreur des batailles (par excellence),

Habile à manier la fronde contre leurs éclatantes murailles (telle) une pique (de sappe),

Tu as dressé ton arme contre la Maison de Kiš.

Tu as terrassé ses 7 héros.

Tu foulas de ton pied la tête d'Enmebaragesi tel un serpent.

Tu es celui qui a rapporté la royauté de Kiš à Uruk/Ur.

60

(Toi) que ... a enfanté, tu as rendu toute sa perfection à Kulaba.

Si Šulgi évoque une guerre menée par Gilgameš contre Kiš, non seulement Uruk n'y apparait pas clairement comme la ville assiégée, mais l'opposant est devenu Enmebaragesi, le père d'Agga. Le sort réservé à ce dernier rappelle la fin du récit d'Utuheg̃al - ainsi d'ailleurs que la captation de la royauté - ce qui laisse supposer que la même tradition inspire ces deux textes. D. Katz envisage que le poète a remplacé, pour les besoins de la gloire de Šulgi, Agga par Enmebaragesi parce que celui-ci était plus fameux. C. Wilcke conjecture que la version de Šulgi repose sur une recension de la Liste royale sumérienne qui faisait se terminer la dynastie de Kiš avec Enmebaragesi. Pourtant l'hymne $\mathrm{O}$ semble faire allusion à un épisode précis lorsqu'il mentionne que sept preux de Kiš ont été vaincus par Gilgameš, alors que ce motif manque totalement dans Gilgameš et Agga. Si tout porte à croire qu'une tradition différente, peut-être plus officielle (O. Edzard, ZA, 53 [1959]), existe vers 2100, la comparaison avec le résumé de l'expédition de Gilgameš contre Huwawa qui suit le passage cité ci-dessus laisse supposer que l'arrangement des aventures de Gilgameš est en partie artificiel dans l'hymne $\mathrm{O}$.

Comparé à Gilgameš et Huwawa A, l'épisode de la guerre avec Kiš a une diffusion beaucoup plus restreinte. Par exemple aucune copie n'a été trouvée à Kiš, Sippar ou Suse. Même le nombre de fragments recueillis à Nippur reste bien inférieur. En outre, ces récits sont parfaitement indépendants et ne contiennent pas de relations explicites entre eux. Il est significatif que le titre de Gilgameš « seigneur de Kulaba » ne figure pas dans Gilgameš et Huwawa A. Seul l'hymne O de Šulgi les relie et les ordonne peut-être chronologiquement. Un catalogue de titres de textes littéraires sumérien (UET VI, 123) range ensemble les deux versions des aventures contre Huwawa (A et B); mais le titre de l'épopée de Gilgameř et Agga ne vient qu'en second lieu (cf. aussi BASOR 8, 12), ce qui suggère qu'aucune relation chronologique et logique précise ne s'imposait.

L'auteur (chantre ou bien scribe inspiré ?) a puisé dans une matière orale et, comme Homère pour l'Iliade, il n'a retenu qu'un épisode bref de l'histoire de la guerre entre Kiš et Uruk. L'histoire commence in medias res (ce qui est néanmoins contesté par T. Marik) avec un ultimatum de la part du roi de Kiš adressé à Gilgameš et sa ville Uruk. Le talent de l'œuvre est de former un tout parfait qui ne rend pas indispensable la connaissance de ce contexte narratif que nous n'avons plus. La présentation du Héros est habilement retardée par un suspense. Après la venue d'une ambassade de Kiš, Gilgameš convoque les Anciens pour leur demander si oui ou non ils veulent se sou- 
mettre à Agga. Face à la décision des Anciens, l'intention et la nature de Gilgameš se révèlent. Il passe outre leur avis, car il est guidé par une légitimation supérieure; son titre n'est d'ailleurs mentionné qu'à partir de ce moment (1. 15-16) :

Bilgames, le seigneur de Kulaba,

Lequel plaçait sa confiance en Inana.

Les Anciens ne font qu'obéir à leur prudence coutumière (c'est un archétype), mais ils peuvent être aveugles aux desseins des dieux. Leur décision va à l'encontre de l'action héroïque. Une des forces du poème est par conséquent de faire confluer deux thèmatiques littéraires opposées, la sagesse respectable mais timorée face au volontarisme épique qui, étant donné le genre, l'emporte. De manière générale, le conflit générationnel est aussi au cœur de l'épopée de Gilgameš (R. Harris, Gender and Aging in Mesopotamia, 2000). Le manque de foi des Anciens dans leurs propres divinités, suggéré par la 1. 16, ne donne pas lieu à un développement car le poème n'est pas théologique et l'auteur s'intéresse plus aux rouages humains! Nous pensons, à la différence de D. Katz (RA, 81 [1987]; LOT 1, 1993), que cela vient du fait que le sujet principal de l'œuvre est l'exaltation de Gilgameš.

Contrairement à la version de l'hymne O de Šulgi, l'agresseur est clairement Agga. Cela donne l'occasion à Gilgameš d'obtenir le titre de roi (ou « chef de guerre » comme le veut Jacobsen), non pas d'Enlil comme le revendique Utu-hegal dans son inscription, mais de l'assemblée politique qui le consacre lugal et ur-sa g̃ (moyennant une petite incohérence puisque la 1.26 le suppose déjà roi, mais le passage est purement «formulaire »: il s'agit d'un proverbe importé dans la matière épique). Le poète continue néanmoins à désigner Gilgameš par son titre traditionnel de en, tandis qu'Agga garde celui de roi, ce qui laisse supposer que le thème « royal » est secondaire. Une lecture unificatrice des traditions sur Gilgameš pourrait faire croire que l'enjeu du poème était l'hégémonie d'Uruk. Mais cette idée n'est nulle part présente explicitement dans le poème. Non seulement la royauté n'est pas offerte par une divinité à Gilgameš, mais les jeunes gens d'Uruk ( $\tilde{\mathrm{g} u r u s ̌})$ ne lui attribuent comme mission que la « possession » d'Uruk, perle des dieux. La fin de l'histoire (après les relectures fondamentales de Jacobsen et Falkenstein) montre que Gilgameš se contente de l'indépendance d'Uruk, mais continue à se désigner comme le subalterne du roi de Kiš.

Le motif de l'ultimatum, répété non sans une certaine pesanteur, serait soit un diktat d'Agga, cherchant à imposer une servitude à Uruk - il s'agirait d'une corvée visant à entretenir les puits ou à pourvoir en eau la ville de Kiš (W. Lambert, Or., 49 [1980], p. 339-340, qui suppose un parallèle avec le livre de Josué), soit une menace d'assécher la nappe phréatique (Y. Wu, NABU 1998/1003). En tous cas, le thème du puits dans la représentation littéraire de la mise en culture de la terre de Sumer est aussi bien illustré par le poème d'Inana et Šukaletuda où l'on apprend qu'une des malédictions suscitées par Inana est l'infection de l'eau des puits par du sang.

La principale action, une fois que le siège d'Uruk a lieu - ce qui ne manque pas de susciter un certain effroi dans Uruk mais de la colère chez les guerriers (W. Römer, TUAT, III/3 [1993], p. 555) - est la venue de Gilgameš sur le rempart. Ce moment est différé par l'apparition préalable au même endroit de son grand-échanson (zabar-dab) que les Kišites prennent d'abord pour le seigneur d'Uruk. Deux sorties sont toutefois 
commandées par Gilgameš avant d'apparaître lui-même devant les ennemis. Dans un premier temps, le guerrier Birhurture se porte volontaire pour aller défier Agga en personne, ce qui au premier abord ressemble à un acte désespéré. Fait prisonnier par les Kišites (1. 61) et conduit devant Agga (1. 63), il délivre une sorte de prophétie avant d'être frappé. Ensuite, le serviteur de Gilgameš, Enkidu engage, seul (dili) le combat dans l'allée de la grand'porte : c'est lui qui révèle apparemment à Agga par un discours identique à celui de Birhurture, que l'homme qui vient de se montrer sur la muraille est cette fois bien Gilgameš en personne. Enkidu serait ainsi encore l'agent des phrases transitives 97-98:

Il lui (Agga) coupa (la retraite vers) les proues des nefs (sur lesquelles il était descendu de Kiš avec son armée).

Il fit prisonnier Agga le roi de Kiš au milieu de sa troupe.

W. Heimpel voit dans le déroulement de ces événements une tactique de Gilgameš (son cheval de Troie!) pour divertir l'attention d'Agga de la grand'porte d'où Birhurture puis Enkidu lancent leur attaque. Si tel est le cas, la déclaration de Birhurture à son chef implique que lui-même n'a pas saisi le plan d'action (1. 57-58) :

Moi, je veux aller contre Agga! (une var. donnerait : [je veux] bondir [contre lui!])

Que sa raison se brouille et qu'il en perde l'esprit!

Cette dernière bravade n'est que l'imitation d'une formule venant de la bouche de Gilgameš (1. 47) qui n'a vraiment de sens que dans la sienne. Ces péripéties (purs artifices?) visent à mettre en valeur le rôle extraordinaire de Gilgameš, ce que préparent déjà les 1. 45-47. Birhurture incarne un officier fanatique de Gilgameš qui sert surtout à révéler le caractère surnaturel de son roi (sinon sa mission serait gratuite); l'action d'Enkidu est le prolongement ou la répétition de la mission accomplie par Birhurture, quoique lui seul, serviteur et bras armé de Gilgameš, fût capable de la mener jusqu'au bout, mais pas sans le concours que crée la diversion de son maître, par le choc de son apparition. Il y a un parallélisme frappant établi entre des paires d'acteurs l'échanson/Gilgameš et Birhurturre/Enkidu, soit l'homme normal comparé au héros : l'effet permet de mettre en valeur la nature surhumaine des héros. Leur modèle est indépassable.

Le rôle de Gilgameš mérite une attention particulière du fait de sa passivité. Mis à part son initiative pour mener la révolte, il n'intervient pas dans le combat personnellement, ce qui n'est pas habituel dans une action épique, il se contente de monter sur le rempart et de s'y pencher. L'effet s'en fait toutefois ressentir immédiatement puisqu'un phénomène qui ressemble à une tempête de poussière se produit :

Par myriades (les uns) se laissèrent tomber, par myriades (les autres) sursautèrent;

Par myriades, ils se « mélangèrent » à la terre;

Le monde entier en fut recouvert;

Même la bouche (des gens) du Pays fut emplie de terre.

La vision fantastique représente peut-être aussi un rite de deuil (attitude hystérique et saupoudrage de poussière); du reste, l'autre fois où Gilgameš se penche ainsi sur la muraille de sa ville, c'est pour y contempler avec horreur des cadavres qui filent sur l'eau (Gilgameš et Huwawa A). Ce passage peut aussi faire penser à ce que Šulgi dit de 
lui-même dans sa correspondance : $u_{17}-l u$-g̃ $u_{10} k a l a m-m a h e_{2}-e b$ - $d u l=$ « Ma tempête est une chape au dessus du Pays! » (P. Michalowski, SBL 3, p. 65). Le seigneur d'Uruk est représenté comme un personnage statique, mais dont la vue provoque la terreur. Bien plus, sa figure est celle d'une icône comme le révèle le discours de Birhurture lors de l'apparition du grand échanson (zabar-dab) qu'Agga prend pour Gilgameš (1. 71-75) :

Cet homme là-bas serait mon roi,

Si son front était écarlate (de colère),

Si ses yeux étaient ceux d'un buffle,

Si sa barbe était de lazulite,

Si son doigt était beau.

Le lien entre le souverain est son grand échanson (ressemblance vestimentaire, même classe sociale ?) est déjà en soi très intéressant car il touche à une réalité du pouvoir (remontant au moins) à l'époque d'Ur III (W. Sallaberger, OBO 160/3, p. 186187) qui se perpétue chez les princes amorrites (M. Guichard, ARM XXXI, p. 43-44). Plus important, cette description fait plus penser à une effigie du héros divinisé qu'à un personnage naturel. L'allusion à l'aspect de son doigt se rapporte ainsi peut-être à un geste royal, signe de communication avec les dieux (M. Herles, AOAT 329, p. 285286 et $C A D$ T' tarāṣu c 1'), figé dans une statue avec placage en or. La déesse de Nagar touche avec le doigt (ina ubānim) celui qu'elle a choisi pour être roi (archives de Šubat-Enlil - Tell Leilān). Cela inspire l'idée que l'application du doigt du roi de Mari (bien vivant) sur un vassal est la marque d'une protection absolue (P. Marello, FM VI, 2002). Or, un culte à Gilgameš est attesté dans divers localités de Sumer (notamment Nippur, Ur et Uruk) à l'époque d'Ur III, mais beaucoup moins bien pour l'époque suivante (T. Richter, AOAT 257, 1999 et A. George, The Babylonian Gilgameš Epic, 2003). Une statue d'un ancêtre aussi puissant (qui a pourtant dû exister à Uruk) pouvait contribuer à la protection de la ville. Le discours d'Utu-hegáal suppose peut-être même un rite d'investiture comprenant un contact direct avec la statue de Gilgameš dont il est le commissionnaire. L'évocation de la venue sur le rempart du héros dans G. et A. pourrait s'inspirer d'un rite dans lequel on faisait sortir la statue de sa cella. La statue de Gilgameš dans sa chapelle (hymne $\mathrm{O}, 43$ ) est considérée comme vivante ce qui justifie toute la scène avec Šulgi que nous avons vue. 11 est vrai que dans ce cas, où les deux héros se parlent d'égal à égal, la scène suppose l'auto-divinisation de Šulgi (P. Michalowski, CSMS Bulletin, 16 [1988] et cf. l'hymne A : 1. 79-80).

L'identification des sources d'inspiration de l'hymne O de Šulgi est, comme on l'a vu, complexe. Nous supposons que son auteur connaissait au moins deux versions concurrentes de $G$. et $A$. et il a joué avec une certaine liberté avec celles-ci permutant les fins de G. et A. (dans la version que nous avons) et Gilgameš et Huwawa. Cependant l'étude de la langue du poème (G. et A.) par C. Wilcke (Mél. Römer) montre à partir de plusieurs observations grammaticales - la réduction du double génitif, le remplacement d'un - $a$ final par $-e$ et des akkadismes tels que l'expression $\breve{s a}_{3}$-erin ${ }_{2}$ na-ka-ne ${ }_{2}$ " au milieu de son armée » (transposition d'une formule akkadienne) - que l'état du texte est récent; il peut avoir été composé au début de l'époque amorrite par un roi d'Uruk. La fonction de Birhurture, $l_{2}$-sag̃-lugal (1. 55), est peut-être aussi un indice de l'origine paléo-babylonienne sinon de la composition entière du moins de la recension que nous avons. 
À partir de toutes ces observations, on peut faire l'hypothèse qu'un précurseur a dû être connu vers 2100 qui lui-même pourrait remonter à l'époque des révoltes contre Naram-Sîn (qui offre un bon contexte rédactionnel). Négligée ou remaniée à l'époque des empereurs d'Ur, un souverain d'Uruk de l'époque amorrite a très bien pu se reconnaître dans l'intrigue de cette vieille histoire et la faire remettre au goût du jour. Quoiqu'une lecture historisante puisse paraître quelque peu réductrice, on peut soupçonner que le texte que nous avons finalement date d'une époque d'éclatement politique et que son rédacteur était un habitant d'Uruk versé dans les traditions anciennes de sa ville, que le souverain pour le compte duquel il travaillait avait pour modèle Gilgameš. C'était un fondateur dynastique fort soucieux de reposer sa légitimité sur une tradition « nationale » qui avait une haute antiquité. Cela pourrait convenir avec ce que l'on sait du roi d'Uruk nommé Sîn-kašid (vers 1860 ; pour ce roi cf. D. Charpin, OBO 160/4, p. 108-109; C. Wilcke suppose cependant une date plus ancienne, vers l'époque de Ballum, Alila-hadûm ou Sumu-banisa), qui se présenta comme « fils de Nin-sumun » (RIME 4.1.1) - il était donc le frère de Gilgameš - restaura l'Eana (RIME 4.1.7) et construisit Ekankal pour Lugalbanda le père de Gilgameš, le tout étant complété d'un gipar pour la prêtresse de Lugalbanda (RIME 4.1.8 et 9). Le vif intérêt des rois d'Uruk pour leurs ancêtres se manifeste encore dans l'inscription d'Anam qui relate les travaux sur la muraille d'Uruk « œuvre ancienne de Gilgameš » (RIME 4.4.6.4). Le regard porté par le « poète » sur le problème de la relation du chef politique avec l'assemblée des Anciens pourrait très bien convenir à un questionnement de la société « amorrite » sur ses institutions, leurs tensions internes et leur évolution (renforcement des pouvoirs monarchiques au détriment des institutions tribales, en l'occurence amnanéennes). Si D. Katz a bien montré le caractère artificiel de l'opposition entre une assemblée des Anciens et celle des jeunes ( $\tilde{\mathrm{g}} u r u s ̌$ ), il faut mentionner que la cohabitation de formes de pouvoirs tels que le « collège » des Anciens avec l'assemblée des gens de la ville (puhrum) ou celle des combattants (pihrum) est bien attestée dans la société amorrite reflétée par les archives de Mari au XVIII ${ }^{\mathrm{e}}$ siècle av. n. è. Enfin, la conclusion du récit n'est pas loin de rappeler une andurārum, chacun retournant à son statut initial (thème présent dans la littérature paléo-babylonienne, cf. par ex. D. Charpin, FM III, 1997).

Reste que l'histoire de ce chef d'œuvre littéraire, le plus politique des récits sur Gilgameš, garde encore son mystère. Ce qui nous est parvenu est une version recueillie par un cercle étroit de lettrés et pourrait être le dernier avatar d'une tradition sumérienne sur Gilgameš en train de devenir désuète, voire déjà morte (malgré une reprise paléo-babylonienne). Les versions à notre disposition attestent un texte relativement bien fixé, mais les nombreuses variantes de détails supposent une certaine liberté laissée au scribe de modifier le détail du texte, ce qui suppose qu'il était utilisé encore au $\mathrm{XVIII}^{\mathrm{e}}$ siècle de manière vivante, comme le montre les jeux sur les modes des verbes (c'est le cas tout particulièrement dans les discours du début) qui, plutôt que des erreurs ou des incertitudes de la tradition, feraient penser à des jeux grammaticaux révélant une des préoccupations des scribes qui manipulaient le poème. L'exemplaire inédit de la collection Schøyen fournit une nouvelle illustration de ces étonnantes variations qui n'affectent ni la structure ni le sens de l'œuvre. 